\title{
Efficient control of transient wave forms to prevent spreading depolarizations
}

\author{
M. A. Dahlem, ${ }^{*}$ F. M. Schneider, and E. Schöll \\ Institut für Theoretische Physik, Technische Universität Berlin, Hardenbergstraße \\ 36, D-10623 Berlin, Germany
}

\begin{abstract}
In various neurological disorders spatio-temporal excitation patterns constitute examples of excitable behavior emerging from pathological pathways. During migraine, seizure, and stroke an initially localized pathological state can temporarily spread indicating a transition from non-excitable to excitable behavior. We investigate these transient wave forms in the generic FitzHugh-Nagumo (FHN) system of excitable media. Our goal is to define an efficient control minimizing the volume of invaded tissue. The general point of such a therapeutic optimization is how to apply control theory in the framework of structures in differential geometry by regarding parameter plane $M$ of the FHN system as a differential manifold endowed with a metric. We suggest to equip $M$ with a metric given by pharmacokinetic-pharmacodynamic models of drug receptor interaction.
\end{abstract}

Key words: nonlinear dynamical system, excitability, control PACS: 05.45.-a

\section{Introduction}

Excitability is a central concept in neurophysiology both in health and disease (Hille, 1997). Under some pathological conditions altered excitability can spread through the tissue. Such transient wave forms (TWF) occur for example in migraine with aura (Lauritzen, 1994; Aurora et al., 1998; Welch, 2005), epilepsy (Somien \& Aitken, 1984; Giorgi et al., 2006), and stroke (Nedergaard \& Astrup, 1986; Hossmann, 1994; Fisher \& Takano, 1995; Hakim, 1998; Back, 1998). In migraine with aura seizure-like activity spreads slowly through parts of the

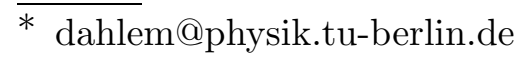

Preprint submitted to Elsevier

10 November 2018 
cortex. This is observed by symptomatic (Lashley, 1941) and electrophysiological (Hadjikhani et al., 2001) events. Likewise, epileptic seizures can have a localized onset and then grow in intensity and start to spread. This usually leads to subsequent generalized motor involvement commonly referred to as partial seizures with secondary generalization. In some cases, however, the epileptiform activity may induce changes to subcortical structures producing clinical signs of general motor involvement that merely mimic a spread (Schindler et al., 2007). Last but not least, during stroke a cortical region that surrounds the infarct core and that initially suffers functional injury can gradually and progressively fail and suffer irreversible structural injury in untreated patients (Baird et al., 1997). In this condition the electrophysiological changes are persistent, but there exist intermediate repetitive TWF and it was suggested that 'therapy might $[. .$.$] target the intermediate forms$ of spreading depolarizations so as to protect the penumbra [tissue surrounding the infarct core] against recruitment into the infarct core' (Dreier et al., 2006). These three paradigmatic clinical manifestations of spreading pathological states motivate efforts to understand how the spread of such states arises and how it can be controlled.

The phenotypical manifestation of such spreading pathological states is the phenomenon of spreading depression (SD) (Leão, 1944). SD is the basis of migraine with aura. Essentially identical electrophysiological features are associated with infarct expansion, called peri-infarct depolarization (PID) (Mayevsky et al., 1996; Strümpl et al., 2002; Fabricius et al., 2006). The cascade of events that produce SD is related to seizures (Kager et al., 2000). Despite certain differences, seizure events that begin with epileptiform discharges can either terminate in SD, facilitate the synchronization, or spread by a similar mechanism over a large area with a velocity resembling that of SD (Bureš et al., 1974; Somjen \& Aitken, 1984; Gorji \& Speckmann, 2004). SD is seizure-like activity evolving as a slowly spreading non-or-all type process. It is characterized by the feedback of ion currents that change ion concentrations, which, in turn, influence the membrane potential (Kager et al., 2000; Somjen, 2001). Shortly after its onset all neuronal activity is depressed, hence its name. The name is misleading, because SD can still be observed even when neuronal activity is depressed by blocking the fast transient sodium current $\mathrm{I}_{N a}$ (Tobiasz \& Nicholson, 1982). SD emerges from an excitable pathway in neuronal tissue independently of the normal neuronal activity. It was therefore suggested to categorize SD and similar phenomena under the term spreading depolarizations (Dreier et al., 2006).

There is ample evidence that SD belongs to the self-organization processes due to the coupling of biochemical reaction with diffusion (Martins-Ferreira et al., 2000; Dahlem \& Chronicle, 2004). Mathematical models of SD have been suggested (Tuckwell \& Miura, 1978; Reggia \& Montgomery, 1994; Shapiro, 2001; Kager et al., 2000; Somjen, 2001), though there is not yet consent on the 
mechanism. We will use the spatially extended FitzHugh-Nagumo (FHN) system as a generic model of neuronal excitation patterns based on reactiondiffusion. As a neuronal model, it describes generic pattern formation properties not limited to nerve impulse propagation along an axon, although it was originally derived from the Hodgkin-Huxley model of action potentials (Hodgkin \& Huxley, 1952; FitzHugh, 1961; Nagumo et al., 1962). The FHN system describes also the spatial features of SD wave in neuronal tissue (Dahlem \& Müller, 2004). Furthermore, the transition from non-excitable to excitable media supporting traveling waves was well investigated in the FHN model (Winfree, 1991; Hakim \& Karma, 1999). It was suggested that the spatio-temporal patterns in SD occur at this transition (Dahlem \& Müller, 2004). The regime in which this transition takes place is also well investigated in chemical model systems in experiment and theory, for a review see (Mikhailov \& Showalter, 2006).

The route to spreading depolarizations in a generic model is provided by two independent pathways: one lowering the threshold of evoked pathological activity, the other changing the time scale of biochemical reaction rates, and hence their time scale ratio. The two pathways might offer new opportunities in developing optimal therapy. Consider the case that a pathological condition is caused by a shift along one path whereas therapeutic strategies are available for both pathways. Suppose both strategies can be combined while each has individual response rates and side effects. What is an optimal time efficient combined therapy stopping the spread while minimizing side effects? Since therapies can be combined, there is a two dimensional manifold in which therapy takes place. The strategy we suggest is to equip this manifold with a metric that allows us to find an effective combined therapy with minimal side effects. Effectiveness is defined by finding a path to a sufficiently low excitability where the tissue is not susceptible to spreading events, whereas efficiency refers to side effects and time.

\section{Parameter space of the FHN system}

We assume that a standard activator-inhibitor scheme leads to the observed propagation phenomena during SD. The activator and inhibitor variables, $u$ and $v$, are coupled by their kinetic reaction rates $f(u, v)$ and $g(u, v)$, respectively, and can diffuse in the medium. The equations are

$$
\begin{aligned}
& \frac{\partial u}{\partial t}=f(u, v)+D_{u} \frac{\partial^{2} u}{\partial x^{2}} \\
& \frac{\partial v}{\partial t}=\epsilon g(u, v)+D_{v} \frac{\partial^{2} v}{\partial x^{2}}
\end{aligned}
$$


Diffusion is represented by diffusion coefficients $D_{u}$ and $D_{v}$. By re-scaling space, the ratio $\delta=\frac{D_{v}}{D_{u}}$ of the diffusion coefficients can be introduced replacing the parameters $D_{u}$ and $D_{v}$. The parameter $\epsilon$ is the time scale ratio of inhibitor and activator variables. The reaction rates $f(u, v)$ and $g(u, v)$ may possibly be derived from a more complex model of SD, e.g., the one from Kager et al. (2000), by lumping together all activator variables, such as inward currents and extracellular potassium concentration $\left[K^{+}\right]_{o}$ into a single activator variable and their combined kinetics into a reaction rate $f(\cdot, \cdot)$. Likewise, a single inhibitor variable could be related to recovery processes, such as effective regulation of $\left[K^{+}\right]_{o}$ by the neuron's $N a-K$ ion pump and the gliaendothelial system. This will be an important task of future investigations. We aim to describe universal features of reaction-diffusion coupling that lead to the onset of spreading pathological states and do not specify the variables $u$ and $v$ which underly these characteristics other than that they play the roles of activator and inhibitor, respectively. Their kinetic functions $f(u, v)$ and $g(u, v)$ are given by the FHN system

$$
\begin{aligned}
& f(u, v)=u-\frac{1}{3} u^{3}-v \\
& g(u, v)=u+\beta-\gamma v
\end{aligned}
$$

where $\beta$ and $\gamma$ determine the excitation threshold (Fig. 1).

We shall start by viewing the parameter plane of the FHN model as a manifold $M$ and consider its geometric structure. $M$ has four dimensions $(\epsilon, \beta, \gamma, \delta)$. In a certain regime the parameters $\beta$ and $\gamma$ determine the threshold for a non-or-all excitation process. Like $\epsilon$, their variation can cause a bifurcation: the emergence of sustained travelling waves. Instead of the four dimensions usually a two dimensional subset is investigated to describe this bifurcation, for example the section at $\gamma=0.5$ (Winfree, 1991), or at $\gamma=0$ (Hakim \& Karma, 1999), both with $\delta=0$. Consider the subset at $\gamma=0$. A particular FHN system is specified by a point $q$ of this subset. It can be parameterized by the coordinate functions $\epsilon(q)$, i. e., the time scale separation of $u$ and $v$, and the threshold parameters $\beta(q)$. As an alternative coordinate function for $\beta(q)$

$$
\Delta(p)=\left(\beta-\frac{1}{3} \beta^{3}\right)
$$

can be chosen (Hakim \& Karma, 1999). While $\beta$ is a measure of the threshold, $\Delta$ is related to a measure of excitability, because it is equal to the inhibitor concentration in the steady state (Fig. 1). This is rather a convention than a definition of excitability. To be more general, we shall only assume that excitability $E$ is a $C^{\infty}$-function $E: U \rightarrow \mathbb{R}$ in a subset $U$ of $M$. Further properties of this function will be defined later. Firstly we want to note that there obviously exists a whole set of coordinate systems $\mathcal{A}$ for $M$ with coordinate 
transformations like Eq. (3) being $C^{\infty}$ diffeomorphisms. Naturally, $M$ can be identified as a $C^{\infty}$ differential manifold. For a given subset $U$ of dimension $n$ we can choose the coordinate system $\xi=\left[\xi^{1}, \ldots, \xi^{n}\right]=\left[\xi^{i}\right] \in \mathcal{A}$, with $i \in\{1,2, \ldots, n\}$ that seems to suit best the purpose of study. For example, in the section with $n=2$ and $\xi^{1}=\epsilon$ the parameter $\xi^{2}$ can be chosen either as $\beta$ or as $\Delta$. We will consider the hypersection at $\delta=0$, and $\gamma=0$ and use various coordinate systems in this submanifold.

\section{Transient Wave Forms (TWF)}

Excitability is an emergent property of active media. It arises when a critical parameter value is crossed above which the medium is susceptible for sustained propagating excitation patterns (Mikhailov \& Zykov, 1991; Winfree, 1991; Hakim \& Karma, 1999; Mihaliuk et al., 2002). In a 1D medium this border in $M$ is the propagation boundary $\partial P$. It is obtained by finding solutions in a co-moving frame in the form $u(x, t)=U(y), v(x, t)=V(y), z=x+c t$, where $c$ is an additional parameter of the unknown wave propagation speed. Travelling wave solutions in the form of a single pulse are thus equivalent to the existence of a homoclinic orbit satisfying a system of ordinary differential equations (Kuznetsov, 1995, p.224 ff). The transition marks a bifurcation of codimension one. Therefore $\partial P$ is a hypersurface in $M$ separating the regime supporting travelling waves from the non-excitable one. In a two-dimensional parameter space, spanned by $\epsilon$, and $\beta$ or $\Delta, \partial P$ is a curve (Fig. 2). Below $\partial P$ any confined perturbation of arbitrary profile decays eventually. Above $\partial P$ some wave profiles are stable and travel with constant velocity.

Consider the region close to $\partial P$, which is referred to as sub-excitable. There transient wave forms (TWF) exist though sustained waves may not. Note first that in the absence of a metric in $M$ closeness to $\partial P$ can only be defined with reference to other borders by which $M$ is further subdivided. The adjacent bifurcation curve above $\partial P$ is $\partial R$. It is only defined in systems with more than one spatial dimension. In these systems $\partial R$ is the border above which open wave fronts will not disappear because each open end curls in to form a spiral. Spiral waves rotate and re-enter multiple times the invaded tissue. Below $\partial R$, but still above $\partial P$, TWF exist depending on the initial size of the excitation. In $2 \mathrm{D}$ such waves occur in the shape of particle-like waves Mihaliuk et al., 2002), which can be controlled by excitability gradients (Sakurai et al., 2002). In this study, we investigate the region below $\partial P$ where unstable TWF exist in the FHN systems with one spatial dimension.

To further subdivide $M$ in the regime below $\partial P$, we suggest to take the distance a TWF spreads (Fig. 3b) to define isolines. This distance defines the volume of tissue at risk (TAR) referring to the risk of transient neurologi- 
cal symptoms or even of permanent damage (PID case) when cortical tissue is invaded following a local stimulation. At $\partial P$ stable traveling waves exist. They can invade the whole tissue. Therefore the TAR value is infinite at $\partial P$. Below $\partial P$ the value of TAR is finite and rapidly decreases to zero when one moves the FHN system away from $\partial P$ in parameter space (Fig. 3b-c). We found the existence of a new boundary $\partial S$ as the TAR value approaches zero. At this boundary any stimulation profile collapses into the steady state without broadening, that is, without affecting surrounding tissue. $\partial S$ is therefore contrary to $\partial P$ at which the TAR value is infinite. The region between $\partial S$ and $\partial P$ is the region where TWF exist in a $1 \mathrm{D}$ system. This region defines a subset $U$ of $M$. The general point of a therapeutic control strategy is to leave $U$ by crossing $\partial S$.

\section{Efficient control of transient wave forms}

So far excitability $E$ has not been defined. We assume $E$ to be a $C^{\infty}$-function in $U$. It is reasonable to assume that $E$ is constant on the two borders, because these are bifurcation lines at which TAR is constant. Furthermore, since a change in TAR is suggestive of a change in excitability, we propose both to be linearly related.

Our goal is to control a path $\Lambda$ in $U$ along which excitability, and with it the volume of TAR, is efficiently diminished. Let the path $\Lambda$ start in $U$ at $q$ where the neuronal tissue temporarily supports the spread of excitation. Let the path end on $\partial S$. Firstly, we assume sufficient knowledge of control characteristics, e.g., by pharmacokinetic and pharmacodynamic means, as described in the next section. We will show in the next section that this provides a metric on $U$. In this section we will just assume $U$ to be a differential manifold with a metric. Furthermore, we assume (i) that our control method allows us to choose any path $\Lambda: I \rightarrow U$ parameterized by some interval $I \subset \mathbb{R}$, (ii), as already stated, that $E$ is a $C^{\infty}$-function in a subset $U$, which includes $\Lambda$, and (iii) that $E(q)>E(\partial S)$ holds.

Which path should we take, if we want to reduce excitability by going from $q$ to $\partial S$ by deliberate control? The efficiency of successful control dragging the system into the target state $p$ on $\partial S$ should be given by some optimization criterion. When a metric is given two paths are privileged: the shortest path $\Lambda_{s}$ between $q$ and $\partial S$, and also the one that minimizes $E$ by gradient descent. The latter path $\Lambda_{g}$ implies a metric because covectors like $\partial E / \partial \xi^{j}$ (representing a gradient) and contravariant vectors (represented by tangent vectors to a path $\Lambda)$ are unrelated objects of different kinds. Only a metric tensor $g_{i j}$ defines 
the gradient as a tangent vector (using summation convention)

$$
(\operatorname{grad} E)^{i}=g^{i j} \frac{\partial E}{\partial \xi^{j}}
$$

Therefore, only when a metric is given on $U$, we can apply some optimization criterion for the efficient therapeutic path.

\section{Metric tensor on $U$}

As efficient control, we proposed in the last section a method based on a metric structure in $U$. Therefore, a metric structure is needed to optimize control. Hence it is natural in this context to endow $U$ with a metric that is derived by some sort of cost function of the control method. We introduce a standard pharmacokinetic and pharmacodynamic scheme to illustrate this concept. Let $\zeta^{i}$ be the concentrations of drugs which regulate diverse functions in populations of neurons. For the sake of simplicity, we neglect the details of pharmacokinetics as the discipline that describes dosage regimes and the time-course of $\zeta^{i}$ in the body by absorption, distribution, metabolism, and excretation. We assume the drugs can be constantly administered and their rate of administrations equals their rate of metabolism and excretion. Thus $\zeta^{i}$ is immediately in its steady state value. Furthermore, we assume that $\zeta^{i}$ follows linear pharmacokinetics. In this situation the steady state of $\zeta^{i}$ changes proportionally according to dose.

The relation between drug dose and response is usually modeled as a hyperbolic function assuming a simple drug receptor interaction. Suppose the response to $\zeta^{i}$ is

$$
\xi^{i}=r^{i}\left(\frac{\xi_{\max }^{i} \zeta^{i}}{E C_{50}^{i}+\zeta^{i}}\right)
$$

where the $\xi^{i}$ define a new coordinate system $\xi=\left[\xi^{1}, \ldots, \xi^{n}\right]$ in $U$. In this equation, $r^{i}$ denotes transducer functions that represent the response of the FHN system to the drug $\zeta^{i}$. For the sake of simplicity we use as transducer functions $r^{i}$ the identity. $E C_{50}^{i}$ are the effective doses 50, i.e., doses at which $50 \%$ of the maximal responses $\xi_{\max }^{i}$ are achieved (Fig. 4a). $\xi_{\max }^{i}$ is the asymptotic value of $\xi^{i}$ for large concentrations.

In analogy with Eq. (3), where we have introduced the new coordinate $\Delta$, we have thus introduced the coordinate system $\zeta \in \mathcal{A}$ as new control parameters

of the FHN system. A new coordinate system with a known metric is needed 
as a reference system from which the metric in $U$ is obtained by coordinate transformation. Let us denote the components of the metric tensor of $U$ in the coordinate system $\zeta$ as $g_{i j}$. Above we have introduced the new coordinate system $\xi \in \mathcal{A}$ of the response variables. The components of the metric tensor in this coordinate system $(\xi)$ are then

$$
\tilde{g}_{\alpha \beta}=\frac{\partial \zeta^{i}}{\partial \xi^{\alpha}} g_{i j} \frac{\partial \zeta^{j}}{\partial \xi^{\beta}}
$$

Likewise, we can calculate the unknown components $g_{i j}$ from $\tilde{g}_{i j}$. The coordinate system $\zeta$ is a privileged reference system for $U$ by selecting $\zeta^{i}$. However, this does not imply that it is Cartesian $\left(\tilde{g}_{i j}=\delta_{i j}, \delta_{i j}\right.$ being the Kronecker delta tensor representing the Euclidean metric in Cartesian coordinates), as is readily seen since the choice of concentration units is arbitrary. For example, commonly the logarithm of the concentration $\zeta^{i}$ is plotted on the abscissa, as shown in Fig. 4a. Hence the question is whether a structure of the pharmacodynamic scheme can be natural choice treated as a metric structure in differential geometry defining $\tilde{g}_{i j}$.

Suppose that $\zeta^{i}$ or one of their metabolites have toxic side effects. Their dose response curve follows the same relation as Eq. (5), although shifted to the right on the dose axis by a higher toxic dose $50\left(T C_{50}\right)$

$$
T^{i}=t^{i}\left(\frac{T_{\max }^{i} \zeta^{i}}{T C_{50}^{i}+\zeta^{i}}\right)
$$

$t^{i}$ denotes transducer functions that represent the response of the toxic system. Again for the sake of simplicity we use as transducer functions $t^{i}$ the identity function. In analogy to Eq. (6) we obtain

$$
\tilde{g}_{\alpha \beta}=\frac{\partial \zeta^{i}}{\partial \xi^{\alpha}} \frac{\partial T^{k}}{\partial \zeta^{i}} \delta_{k l} \frac{\partial T^{l}}{\partial \zeta^{j}} \frac{\partial \zeta^{j}}{\partial \xi^{\beta}},
$$

assuming $T=\left[T^{1}, \ldots, T^{n}\right]$ builds a Cartesian coordinate system of the costs with the Euclidean metric $\delta_{k l}$. The association of the cost coordinate system $(T)$ with the Cartesian one is a naturally choice, since the efficiency of control is-by definition-measured in these costs.

We end this section with one example that to some extent corresponds to a subtype of migraine with aura. For this subtype a novel pathogenic genetic mutation was found. Therefore, at least in principle, the route to hyperexcitability was discovered on a molecular level. A recent migraine study by Vanmolkot et al. (2007) showed a mutation in the gene coding the sodium 
channel that leads to a slowed-down inactivation and a two-fold faster recovery from inactivation. In our simplified FHN system this is modeled by slowed inhibition, i. e., lower $\epsilon$ values. As a consequence, the physiological state ( $\mathbf{\Lambda}$, Fig. 4d) of the cortex is shifted into hyperexcitable state (ם). By definition, the physiological state $(\mathbf{\Lambda})$ is on a lower TAR-level than the hyperexcitable state (匹). The therapeutic goal would be to shift the cortical state back to low a TAR-level, at which the pathological activity, i. e., the neurological symptoms, do not spread.

To begin with, we need to specify dose response and toxicity curves. They are chosen arbitrarily to complete the example but without direct relation to the above mentioned subtype of migraine. Suppose that we have a drug with concentration $\zeta^{1}$ acting exclusively on $\epsilon$ (e. g., $\left.\xi^{1} \equiv \epsilon\right)$. Its dose response graph is shown in Fig. 4b. It is similar to the one in Fig. 4a but rotated such that the response axis corresponds to the $\epsilon$-axis. Let another $\operatorname{drug} \zeta^{2}$ act exclusively on $\beta$ (e. g., $\xi^{2} \equiv \beta$ ). Its dose response graph is shown in Fig. 4 c. It is mirrored (from Fig. 4a) such that the response axis corresponds to the $\beta$-axis. For the sake of readability we replace the set $\{1,2\}$ of indices $i$ by $\{\epsilon, \beta\}$ and use $\zeta^{i}$ for both the drug concentration and the drug name. Let the effective doses 50 $E C_{50}^{i}$ be the same for both (without loss of generality we choose $E C_{50}^{i}=0.1$ ), and let the toxic doses $50 T C_{50}^{i}$ differ. Usually $T C_{50}^{i}$ is several times higher then the effective dose. We choose $T C_{50}^{\epsilon}=5$ and $T C_{50}^{\beta}=10$. The drug $\zeta^{\beta}$ has therefore a relatively higher therapeutic index $T I^{i}=T C_{50}^{i} / E C_{50}^{i}$ than $\zeta^{\epsilon}$. Furthermore, we assume that both drugs have a sufficient efficacy, that is, they are each potent enough to drag the FHN system back to it physiological excitability defined by the TAR-isoline (Fig. 4d). The maximal response rates $\left(\xi_{\max }^{i}\right)$ for both are chosen such that already $80 \%$ of $\xi_{\max }^{i}$ is effective. Likewise and again without loss of generality we fix $T_{\max }^{i}$ at $\xi_{\max }^{i}$.

Given the example described above, we can calculate the cost for individual and combined therapy. If exclusively $\operatorname{drug} \zeta^{\beta}$ is administered to control excitability, the FHN system moves in parameter space along the $\beta$-axis (downwards in Fig. 4d) until the physiological TAR level (TAR low) is reached. The toxicity imposed by the therapeutic benefit in this case is $3.85 \%$ of the maximal toxic level of $T^{\beta}$. An exclusive administration of $\operatorname{drug} \zeta^{\epsilon}$ (which has a only half the therapeutic index $T I^{\epsilon}$ compared to $T I^{\beta}$ ) cost $7.4 \%$ of the maximal toxic level $T^{\epsilon}$. This corresponds to a shift from point marked with $\mathbf{\square}$ to the point marked with $\boldsymbol{\Delta}$ in Fig. 4d. At last, we consider how the two drugs must be administered in a combined therapy. An optimal combined therapy reaches the physiological TAR level at $1.84 \%$ of the maximal combined toxic level $T_{\text {tot }}=T^{\epsilon}+T^{\beta}$ (see the two black lines starting at $(\bullet)$ and terminating at the both effect axes in Fig. 4b and d). Due to the nonlinearity in the response and toxcity curves the total cost of the combined theraby is much less than the average of the individual therapies. 


\section{Discussion}

The phenotypical scheme of cortical spreading depression describes transient waves of massive depolarization of neurons and astrocytes. Such a spreading depolarization (Dreier et al., 2006) is associated with migraine, epilepsy and stroke. Their etiologies are mainly discussed in the context of hyperexcitability and disorders known as channelopathies, that is, diseases caused by a mutation in gene coding for ion channels. The challenge is to bridge the gap between the molecular level of the cause and macroscopic tissue level of the effects.

We suggest that there are at least two independed routes towards a hyperexcitable state that supports transient wave forms in cortical tissue: one changing the ratio of kinetic rates and one lowering the threshold. While the latter route changes the nullclines, the former changes only the trajectories in the phase space. Consequently, there are also two routes out of the hyperexcitable regime. This is of particular interest when a critical therapeutic time window exits in which the volume of affected tissue is largely increased, as for example in peri-infarct depolarization. Then the therapeutic aim would be first to prevent tissue loss within the given therapeutic time window and re-establish the physiological value later. In Fig. $4 \mathrm{~d}$ this would correspond to a path from the hyperexcitable state $(\boldsymbol{\square})$ directly to a state of low tissue at risk $(\bullet)$ and from there back to the physiological state $(\mathbf{\Delta})$.

We have described efficient control of excitability by a simple pharmacodynamic model. In general, the study of beneficial effects of independent pathways is complicated by numerous interactions between pharmacokinetics, pharmacodynamics, and homeostatic factors and by individual variability. For example, the introduction of antagonistic behavior between $\zeta^{\epsilon}$ and $\zeta^{\beta}$ will complicate the geometrical structure of $U$. In general the coordinate system of the costs $\left[T^{1}, \ldots, T^{n}\right]$ will not build a Cartesian coordinate system. However, if we have a mapping from general costs and effects defined by Eqs. (5) and (7) we still can infer a metric structure of the parameter space of FHN.

In conclusion, as a first step to implement the proposed control strategy accurate estimates given by pharmacokinetic-pharmacodynamic models of drug activity are needed for the regime of transient wave forms. We want to stress this point, namely that this estimates must be obtained in the regime of transient wave forms, that is, a regime close to a bifurcation. Attempts to obtain estimates of pharmacokinetic-pharmacodynamic parameters in the regime far from this bifurcation will probably lead to inaccurate results. Then the next step is to construct an appropriate metric on the differential manifold of the parameter space in order to optimize the path from the hyperexcitable state back to the physiological state. 


\section{Acknowledgments}

We are grateful to G. Bordyugov, and V. Zykov for fruitful discussions. MAD would like to thank the Deutsche Forschungsgemeinschaft for financial support (DA-602/1-1). This work was also supported within the framework of Sfb 555.

\section{References}

Aurora, S. K., Ahmad, B. K., Welch, K. M., Bhardhwaj, P. \& Ramadan, N. M. [1998] "Transcranial magnetic stimulation confirms hyperexcitability of occipital cortex in migraine," Neurology 50, 1111.

Back, T. [1998] "Pathophysiology of the ischemic penumbra-revision of a concept," Cell. Mol. Neurobiol. 18, 621.

Baird, A. E., Benfield, A., Schlaug, G., Siewert, B., Lovblad, K. O., Edelman, R. R. \& Warach, S. [1997] "Enlargement of human cerebral ischemic lesion volumes measured by diffusion-weighted magnetic resonance imaging," Ann. Neurol. 41, 581.

Bureš, J., Burešova, O. \& Křivănek, J. [1974] The mechanism and applications of Leão's Spreading Depression (Academia, New York).

Dahlem, M. A. \& Chronicle, E. P. [2004] "A computational perspective on migraine aura," Prog. Neurobiol. 74(6), 351.

Dahlem, M. A. \& Müller, S. C. [2004] "Reaction-diffusion waves in neuronal tissue and the window of cortical excitability," Ann. Phys. 13(7), 442.

Dreier, J. P., Woitzik, J., Fabricius, M., Bhatia, R., Major, S., Drenckhahn, C., Lehmann, T.-N., Sarrafzadeh, A., Willumsen, L., Hartings, J. A., Sakowitz, O. W., Seemann, J. H. et al. [2006] "Delayed ischaemic neurological deficits after subarachnoid haemorrhage are associated with clusters of spreading depolarizations," Brain 129, 3224.

Fabricius, M., Fuhr, S., Bhatia, R., Boutelle, M., Hashemi, P., Strong, A. J. \& Lauritzen, M. [2006] "Cortical spreading depression and peri-infarct depolarization in acutely injured human cerebral cortex," Brain 129, 778.

Fisher, M. \& Takano, K. [1995] "The penumbra, therapeutic time window and acute ischaemic stroke," Baillieres Clin. Neurol. 4, 279.

FitzHugh, R. [1961] "Impulses and physiological states in theoretical models of nerve membrane," Biophys. J. 1, 445.

Giorgi, F. S., Lazzeri, G., Natale, G., Iudice, A., Ruggieri, S., Paparelli, A., Murri, L. \& Fornai, F. [2006] "MDMA and seizures: a dangerous liaison?," Ann. N. Y. Acad. Sci. 1074, 357.

Gorji, A. \& Speckmann, E.-J. [2004] "Spreading depression enhances the spontaneous epileptiform activity in human neocortical tissues," Eur. J. Neurosci. 19, 3371.

Hadjikhani, N., Sanchez Del. Rio., M., Wu, O., Schwartz, D., Bakker, D., Fis- 
chl, B., Kwong, K. K., Cutrer, F. M., Rosen, B. R., Tootell, R. B., Sorensen, A. G. \& Moskowitz, M. A. [2001] "Mechanisms of migraine aura revealed by functional MRI in human visual cortex," Proc. Natl. Acad. Sci. USA 98, 4687.

Hakim, A. M. [1998] "Ischemic penumbra: the therapeutic window," Neurology $\mathbf{5 1}(3$ Suppl 3), 44.

Hakim, V. \& Karma, A. [1999] "Theory of spiral wave dynamics in weakly excitable media: asymptotic reduction to a kinematic model and applications," Phys. Rev. E 60, 5073.

Hille, B. [1997] Ionic Channels of Excitable Membranes (Sinauer As).

Hodgkin, A. L. \& Huxley, A. F. [1952] "A Quantitative Description of Membrane Current and its Application to Conduction and Excitation in Nerve," J. Physiol. 117, 500.

Hossmann, K. A. [1994] "Viability thresholds and the penumbra of focal ischemia," Ann. Neurol. 36, 557.

Kager, H., Wadman, W. J. \& Somjen, G. G. [2000] "Simulated seizures and spreading depression in a neuron model incorporating interstitial space and ion concentrations," J. Neurophysiol. 84, 495.

Kuznetsov, Y. A. [1995] Elements of Applied Bifurcation Theory (Springer, New York).

Lashley, K. [1941] "Patterns of Cerebral Integration inicated by Scotomas of Migraine," Arch. Neurol. Psychiatry 46, 331.

Lauritzen, M. [1994] "Pathophysiology of the migraine aura. The spreading depression theory [see comments]," Brain 117, 199.

Leão, A. A. P. [1944] "Spreading depression of activity in the cerebral cortex," J. Neurophysiol. 7, 359 .

Martins-Ferreira, H., Nedergaard, M. \& Nicholson, C. [2000] "Perspectives on spreading depression," Brain. Res. Brain Res. Rev. 32(1), 215.

Mayevsky, A., Doron, A., Manor, T., Meilin, S., Zarchin, N. \& Ouaknine, G. E. [1996] "Cortical spreading depression recorded from the human brain using a multiparametric monitoring system," Brain Res. 740, 268.

Mihaliuk, E., Sakurai, T., Chirila, F. \& Showalter, K. [2002] "Feedback stabilization of unstable propagating waves," Phys. Rev. E 65(6), 065602.

Mikhailov, A. S. \& Showalter, K. [2006] "Control of waves, patterns and turbulence in chemical systems," Phys. Rep. 425, 79.

Mikhailov, A. S. \& Zykov, V. S. [1991] "Kinematical theory of spiral waves in excitable media: comparison with numerical simulations," Physica D 52, 379.

Nagumo, J., Arimoto, S. \& Yoshizawa., S. [1962] "An active pulse transmission line simulating nerve axon.," Proc. IRE 50, 2061.

Nedergaard, M. \& Astrup, J. [1986] "Infarct rim: effect of hyperglycemia on direct current potential and [14C]2-deoxyglucose phosphorylation," J. Cereb. Blood Flow Metab. 6, 607.

Reggia, J. A. \& Montgomery, D. [1994] "Modeling cortical spreading depression," Proc. Annu. Symp. Comput. Appl. Med. Care, 873. 
Sakurai, T., Mihaliuk, E., Chirila, F. \& Showalter, K. [2002] "Design and control of wave propagation patterns in excitable media," Science 296, 2009.

Schindler, K. A., Leung, H., Lehnertz, K. \& Elger, C. E. [2007] "How generalized are secondarily "generalized" tonic-clonic seizures?," J Neurol Neurosurg Psychiatry .

Shapiro, B. E. [2001] "Osmotic forces and gap junctions in spreading depression: a computational model," J. Comput. Neurosci. 10, 99.

Somjen, G. G. [2001] "Mechanisms of spreading depression and hypoxic spreading depression-like depolarization," Physiol. Rev. 81, 1065.

Somjen, G. G. \& Aitken, P. G. [1984] "The ionic and metabolic responses associated with neuronal depression of Leao's type in cerebral cortex and in hippocampal formation," An. Acad. Bras. Cienc. 56(4), 495.

Strümpel, C., Astrov, Y. A. \& Purwins., H. G. [2002] "Multioscillatory patterns in a hybrid semiconductor gas-discharge system.," Phys. Rev. E 65, 066210.

Tobiasz, C. \& Nicholson, C. [1982] "Tetrodotoxin resistant propagation and extracellular sodium changes during spreading depression in rat cerebellum," Brain Res. 241, 329.

Tuckwell, H. C. \& Miura, R. M. [1978] "A mathematical model for spreading cortical depression," Biophysical J. 23, 257.

Vanmolkot, K. R. J., Babini, E., de Vries, B., Stam, A. H., Freilinger, T., Terwindt, G. M., Norris, L., Haan, J., Frants, R. R., Ramadan, N. M., Ferrari, M. D., Pusch, M. et al. [2007] "The novel p.L1649Q mutation in the SCN1A epilepsy gene is associated with familial hemiplegic migraine: genetic and functional studies.," Hum. Mutat. 28, 522.

Welch, K. M. [2005] "Brain hyperexcitability: the basis for antiepileptic drugs in migraine prevention," Headache 45, 25.

Winfree, A. T. [1991] "Varieties of Spiral Wave Behaviour: An experimentalist's approach to the theory of excitable media," Chaos 1, 303. 


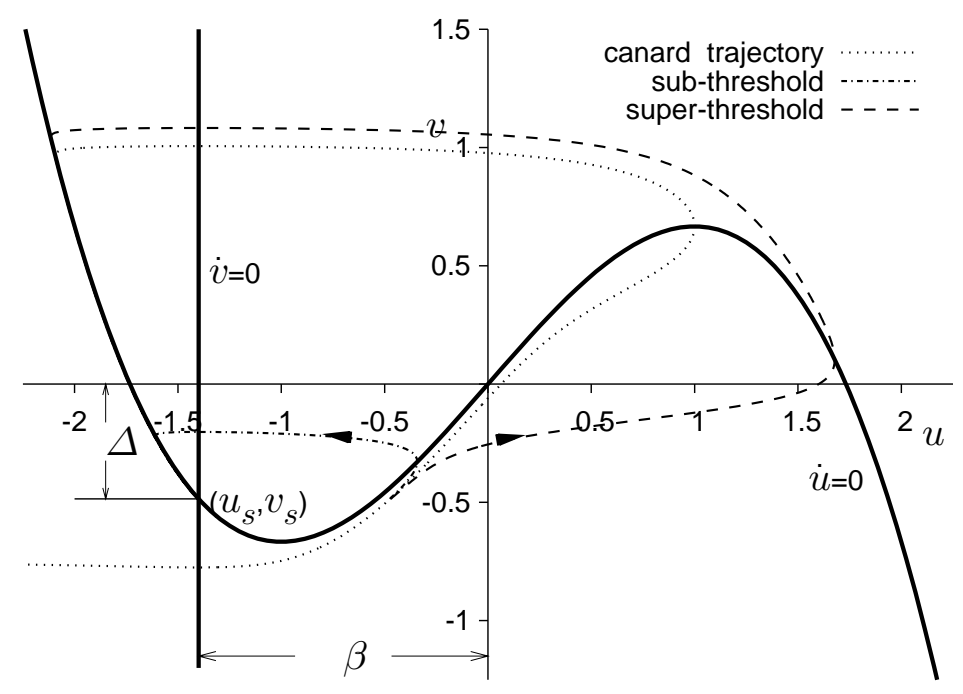

Fig. 1. The nullclines (bold) $\dot{u}=0$ and $\dot{v}=0$ in the phase space of the homogeneous FHN system with $\gamma=0, \beta=1.4$. Their intersection at $\left(u_{s}, v_{s}\right)$ is a stable fixed point. Three trajectories are drawn for $\epsilon=0.04$ : one canard trajectory (dotted), passing through the maximum of the nullisocline $\dot{u}=0$, and two trajectories starting at $v=v_{s}$ nearby but on opposite sides of the canard trajectory. They diverge sharply, producing threshold behavior: (dashed) $=$ super-threshold and (dashed-dotted) $=$ sub-threshold stimulation. The parameter $\beta$ correlates with the threshold size, while $\Delta$ is in a certain range inversely related and therefore correlates with the excitability of the system (see text). 

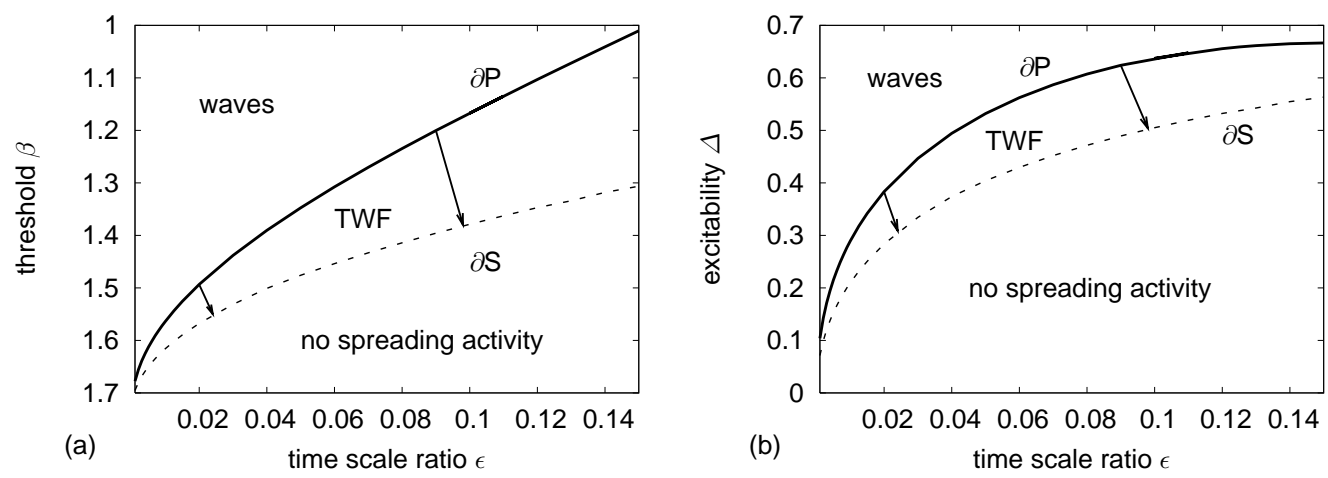

Fig. 2. Parameter space of the FHN system $(\gamma=0, \delta=0)$. Besides the parameter $\epsilon$, a threshold parameter $\beta$ and an excitability parameter $\Delta$ is used in (a) and (b), respectively, to span the parameter space (see Fig. 1). Three regimes exist defined by the spatio-temporal patterns that occur: waves, transient wave forms (TWF) and no spreading activity. The arrows mark two paths which are perpendicular to the border $\partial S$ in (a) but not in (b). 

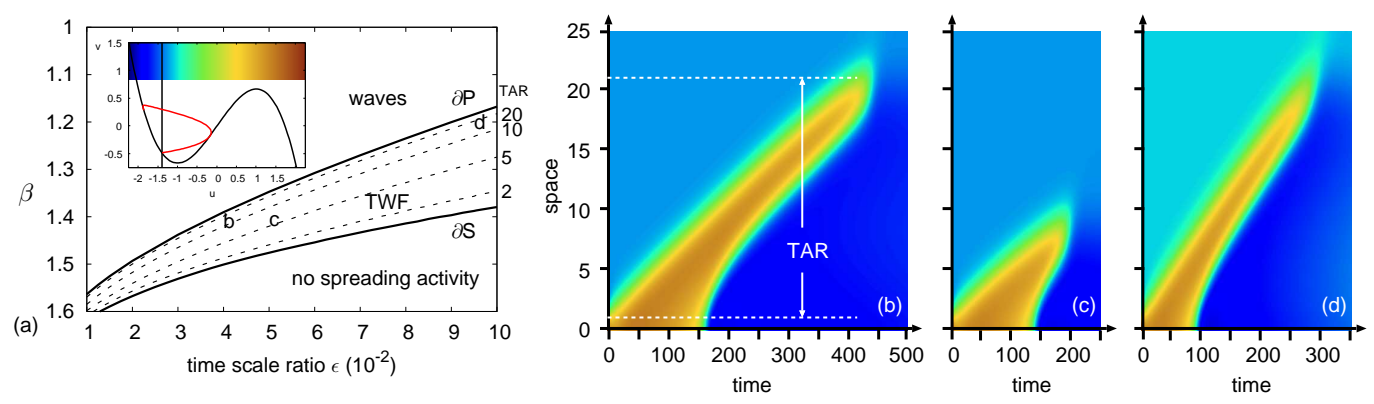

Fig. 3. (a) Parameter space with tissue-at-risk(TAR)-isolines (dashed). The extent a TWF spreads is defined from the stimulation border to the location where the maximum activator concentration $u$ lies above the nullisocline $\dot{u}=0$ (inset). The red curve in the inset is the projection of a TWF from the infinite phase space of the space-dependent FHN system into the one of the homogeneous system for $\beta=1.4, \epsilon=0.04$. The projection is taken at the moment which we defined as the collapse of the TWF. (b)-(d) Space-time plots of the transient wave forms (TWF) following a stimulation (increase of $u$ by 2 for $0<x<1$, starting from the fixed point). Parameters: (b) $\epsilon=0.04, \beta=1.4$, (c) $\epsilon=0.05, \beta=1.4$, (d) $\epsilon=0.095, \beta=1.185 . D_{u}=1$ and $\delta=0$ for all parts. The color code denotes the activator $u$ as defined in the inset of panel (a). 

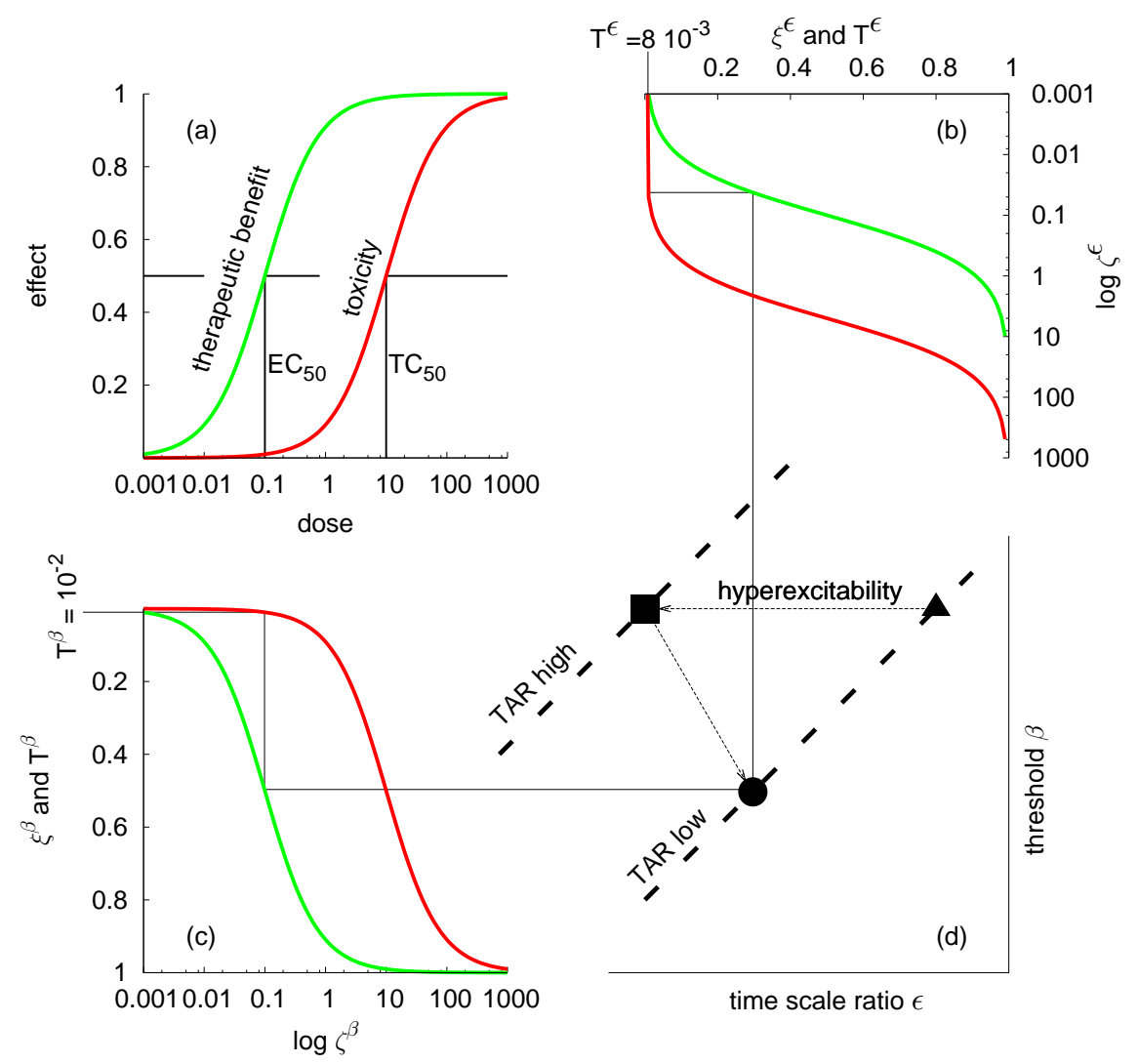

Fig. 4. (a) Dose response curves for an effective dose and a toxic dose with the typical sigmoidal shape caused by the logarithmic scaling. (b),(c) Same as in (a), but with rotated and inverted axes for two drugs $\zeta^{\epsilon}$ acting on $\epsilon$ and $\zeta^{\beta}$ acting on $\beta$. (d) Schematic parameter space with two TAR-isolines (TAR high and TAR low). A route to hyperexcitability can be caused by a shift in $\epsilon$ (from $\boldsymbol{\Delta}$ to $\mathbf{\square}$ ). The optimum path from the hyperexcitable state $(\boldsymbol{\square})$ towards the isoline of the physiological state (TAR low) using the two dose and toxic response curves in (b) and (c) leads to (•). 